\title{
Penile prosthesis implantation in Chinese patients with severe erectile dysfunction: 10-year experience
}

\author{
Wei-Dong Song ${ }^{1 *}$, Yi-Ming Yuan ${ }^{1 *}$, Wan-Shou Cui ${ }^{1}$, Alex $\mathrm{K} \mathrm{Wu}^{3}$, Yi-Chen $\mathrm{Zhu}^{1,2}$, Jing Liu ${ }^{1}$, Lin Wang ${ }^{1}$, \\ Guang-Yi Bai ${ }^{1}$, Jing Peng ${ }^{1}$, Zhi-Chao Zhang ${ }^{1}$, Bing Gao ${ }^{1}$, Ying-Lu Guo ${ }^{1}$, Tom F Lue ${ }^{3}$ and Zhong-Cheng Xin ${ }^{1}$
}

We retrospectively evaluated the clinical outcome of penile prosthesis implantation (PPI) in Chinese patients with severe erectile dysfunction (SED). From July 2000 to December 2011, 224 patients (mean age: 35.9 \pm 11.8 years, range: 20-75 years) with SED underwent PPI by experienced surgeon according to standard PPI procedure at our centre. A malleable prosthesis (AMS 650) was implanted in 45 cases (20.1\%), and a three-piece inflatable prosthesis (AMS 700 CXM or AMS 700 CXR) was implanted in 179 cases (79.9\%). Surgical outcomes, including postoperative complications, clinical efficacy and couple satisfaction, were evaluated over than 6 months postoperatively using medical record abstraction, IIEF-5, quality of life (QoL) scores, and the patient/partner sexual satisfaction score proposed by Bhojwani et al. Of the 224 patients eligible for the study, 201 subjects (89.7\%) completed follow-up. All of patients could perform sexual intercourse post PPI with the mean postoperative IIEF-5 and QoL scores were 20.02 \pm 2.32 and $5.28 \pm 0.76$, respectively, which were significantly improved compared with the preoperative scores $(6.29 \pm 1.5$ and $2.13 \pm 0.84$, $P<0.01)$. Of the 201 men, mechanical malfunction occurred in four cases $(2.0 \%)$ and three cases were re-implanted new device, and two cases $(1.0 \%)$ developed a mild curvature of the penis. Scrotal erosion with infection occurred in one case with diabetes mellitus $(0.5 \%)$ and required complete removal of the implanted AMS 700 CXM. Satisfactory sexual intercourse at least twice per month was reported by 178 men $(88.6 \%$ ), and overall satisfaction with the PPI surgery was reported by $89.0 \%$ of men and $82.5 \%$ of partners. Patient satisfaction in the three-piece inflatable prosthesis group was higher than in the malleable prosthesis group $(P<0.05)$.

Satisfaction, however, between the types of prostheses, did not differ in the partner survey. PPI is a safe and effective treatment option for Chinese patients with SED and experienced surgeon perform PPI according to standard PPI procedure could reduce the postoperative complications of PPI and could improve patient satisfaction ratio and QoL.

Asian Journal of Andrology (2013) 15, 658-661; doi:10.1038/aja.2013.78; published online 22 July 2013

Keywords: complication; erectile dysfunction (ED); implantation; quality of life (QoL); penile prosthesis; satisfaction

\section{INTRODUCTION}

Erectile dysfunction (ED) is defined as 'the consistent inability to achieve and maintain a penile erection adequate for satisfactory sexual intercourse'. ${ }^{1}$ ED may substantially decrease the quality of life (QoL) for both the man and his partner. ${ }^{1}$ The reported prevalence of $\mathrm{ED}$ is greater than $50 \%$ in men over the age of 50 years and likely affects millions of men in China. ${ }^{2} \mathrm{ED}$ may be related to vascular disease, neurological disease, hormonal defects, cavernosal fibrosis from Peyronie's disease or ischaemic priapism, or diabetes mellitus. ${ }^{2}$

The current first-line treatment for ED is the oral administration of a phosphodiesterase type $\mathrm{V}$ inhibitor. The phosphodiesterase type $\mathrm{V}$ inhibitor has revolutionized ED management due to the inhibitor's high rates of efficacy and favourable adverse-effects profile. ${ }^{3}$ Secondline treatment options include intracavernous injection therapy with papaverine, phentolamine or prostaglandin E1, given separately or as combination therapy. The clinical efficacy of intracavernous injection therapy is high, but inconvenience, pain and the risk of ischaemic priapism slightly limit the utilisation of this treatment. ${ }^{4}$ Vacuum tumescence devices may also be used as second-line therapy and are effective in certain men.

In patients who do not respond to the medical management of $\mathrm{ED}$, penile prosthesis implantation (PPI) is a definitive option for $\mathrm{ED}$ management. Modern penile prostheses have been available since the early 1970s. Continuous refinements in the devices and surgical techniques for placement have made PPI a highly effective management strategy for refractory ED. ${ }^{5}$

Penile prosthetic devices were approved by the Chinese State Food and Drug administration in 2000; despite their approval, high cost and poor patient acceptance have limited the use of PPI in China. However, the high rate of economic development in China has led many Chinese men to seek treatment for ED. Although the cost continues to be prohibitive for many Chinese men, PPI is gaining acceptance in China as a management option for ED. Long-term data on outcomes, however, are lacking.

Our Andrology centre has been performing PPI by experienced surgeon according to standard PPI procedure for over 10 years. 
During that time, we have performed 224 PPIs, which is by far the largest number at a single Chinese centre. In this manuscript, we report the outcomes.

\section{MATERIALS AND METHODS}

We retrospectively reviewed medical records from July 2000 (the time of approval of penile prosthetic devices in China) to December 2011 to identify men who had undergone penile prosthesis placement at our institution, following approval from the Peking University First Hospital Institutional Review Board. The indications for PPI were as follows: (i) severe erectile dysfunction (SED); (ii) failure or intolerance of medical management; and (iii) the confirmation of organic ED by special evaluation, such as Doppler ultrasound or cavernosography. Informed consent was obtained from patients with or without their spouses present. Patients with psychiatric illness, genital or systemic infections, perineal wounds, severe liver and kidney dysfunction, and/ or uncontrolled diabetes or hypertension were not considered as candidates for PPI.

All patients underwent a routine preoperative examination including testing for serum electrolytes and clotting factors, an electrocardiography, a chest X-ray and a urine culture to confirm that no surgical contraindications existed. The advantages and disadvantages of each device available on the Chinese market were discussed with all patients. The surgical technique was based on the standard PPI procedure and our early experience. ${ }^{5,6}$ During the operation, the operative field and surgeon's hands were disinfected for more than $15 \mathrm{~min}$ with a povidone-iodine scrub. Three-piece inflatable prostheses were implanted through a transverse penoscrotal incision, and malleable prostheses were implanted through a distal penis shaft incision. Antibiotic prophylaxis, such as vancomycin, was administered preoperatively and was continued for 3-5 days postoperatively. The implant and corpus cavernosa were irrigated with antibiotic saline prior to implantation, and a scrotal vacuum drain was placed to minimize postoperative scrotal haematoma formation. Most of the patients undergoing surgery received spinal anaesthesia, and nine cases $(4.0 \%)$ received general anaesthesia due to contraindications for spinal anaesthesia. Patients were admitted to the hospital 3-5 days postoperatively.

In several cases, adjunctive surgical procedures were performed, including in patients who had urethral strictures and underwent direct vision internal urethrotomy at the time of $\mathrm{PPI}^{7}$ and in patients with severe fibrosis of the corpus cavernosa due to ischaemic priapism who underwent extensive resection of the scar tissue, followed by corporal reconstruction.

Patients were taught how to operate the prostheses after surgery and were allowed to begin sexual intercourse 6 weeks after PPI surgery. A follow-up questionnaire was administered to the patients by letter, telephone or in person. The survey was based on published literature, ${ }^{7-10}$ with several modifications to account for Chinese cultural norms and expectations. The questionnaire consisted of four parts, including an assessment of erectile function with the IIEF-5, a QoL question ('how would you feel if you were to spend the rest of your life with your erectile function the way it is now', rated on a 0-6 scale, from 'terrible' to 'delighted'), an assessment of the frequency of sexual activity/device usage and a patient/partner sexual satisfaction score proposed by Bhojwani et al. ${ }^{8}$ (a score from 1 to 10 is given to this therapeutic method, with a score greater than or equal to 6 identified as satisfaction and less than or equal to 5 identified as dissatisfaction). Data analysis was performed using SPSS (Statistical Package for the Social Sciences) v. 13.0 statistical software (Chicago, IL, USA), and statistical significance was determined using the rank-sum test for continuous data and the Chi-square test for categorical data, with $P<0.05$ indicating statistical significance.

\section{RESULTS}

PPI was performed successfully in 224 men at our centre by the experienced surgeon in the period between June 2000 and December 2011. The mean age of patients was $35.9 \pm 11.8$ years (range: $20-75$ years). The diseases contributing to the patients' ED are shown in Table 1. The most common causes of ED in our study population were as follows: vasculogenic (57.6\%), neurogenic (15.2\%), traumatic from pelvic fracture (11.6\%), diabetic (4.0\%), Peyronie's disease $(4.0 \%)$ and fibrosis from ischaemic priapism $(4.0 \%)$. The average length of the left cavernosum was $16.0 \pm 1.5 \mathrm{~cm}$ and of the right cavernosum was $16.1 \pm 1.5 \mathrm{~cm}$. Of the prostheses inserted, $45(20.1 \%)$ were malleable prostheses (American Medical Systems AMS 650), and 179 (79.9\%) were three-piece inflatable prostheses (168 AMS 700 CXM and 11 AMS 700 CXR). The type of prosthesis used was based on patient choice. In total, 106 cases $(47.3 \%)$ were primary ED, marked by suffering from ED since the first attempt at sexual intercourse, ${ }^{10}$ and 118 cases were secondary ED. In this study, 16 men with ED secondary to pelvic fracture also had urethral stricture and underwent simultaneous PPI and direct vision internal urethrotomy. The nine patients with severe corporal fibrotic scarring due to ischaemic priapism underwent extensive resection of the scar tissue, followed by corporal reconstruction.

A total of 201 men (89.7\%) were available for follow-up evaluation, with a mean follow-up time of $68.3 \pm 36.3$ (range: $6-133$ ) months. Of these patients, an AMS 650 was utilized in 40 cases, an AMS 700 CXM was used in 150 cases and an AMS 700 CXR was implanted in 11 cases that were followed up. The remaining 23 cases were lost to follow-up due to patients' changed telephone numbers.

Mechanical failure occurred in four cases $(2.0 \%)$ of implanted AMS 700 CXM (fluid leakage in two, pump auto-inflation in two) at a mean of 19 (10-31) months postoperatively. Scrotal erosion with infection occurred in one patient with diabetes mellitus $(0.5 \%)$ after 36 months. At the time of follow-up, 178 patients $(88.6 \%)$ continued to have regular sexual activity more than twice per month. There was no significant difference in the rate of sexual activity between the threepiece inflatable prosthesis group and the malleable prosthesis group $(P>0.05)$. In the remaining 23 cases $(11.4 \%)$, sexual activity was irregular, which was attributed to spousal factors (health or divorce). Two patients (1.0\%) with neurogenic ED reported the impairment of ejaculation and orgasm after PPI. No other patients reported changes in ejaculation or orgasm.

Table 1 Primary aetiology of severe erectile dysfunction in 224 patients with implanted penile prostheses

\begin{tabular}{lc}
\hline Primary aetiology & $\mathrm{n}(\%)$ \\
\hline Vasculogenic & $129(57.6)$ \\
Neurogenic & $34(15.2)$ \\
Pelvic trauma & $26(11.6)$ \\
Diabetes mellitus & $9(4.0)$ \\
Peyronie's disease & $9(4.0)$ \\
Ischaemic priapism & $9(4.0)$ \\
Spinal cord injury & $2(0.9)$ \\
Psychogenic & $2(0.9)$ \\
Unknown & $4(1.8)$ \\
Total & $224(100)$ \\
\hline
\end{tabular}


Over than 6 months post-surgery, 201 patients completed evaluation of the sexual function and QoL questionnaires. All of patients could perform sexual regular intercourse with the mean IIEF- 5 scores significantly improved from $6.29 \pm 1.54$ (preoperative) to $20.02 \pm 2.32$ (postoperative) $(P<0.01)$. Postoperative QoL scores also significantly increased from $2.13 \pm 0.84$ to $5.28 \pm 0.76 \quad(P<0.01)$. Based on Bhojwani's sexual satisfaction score, the overall satisfaction rate for the PPI surgery was $89.0 \%$ for men and $82.5 \%$ for their partners. The results are summarized in Table 2 . Patient satisfaction was significantly greater among those individuals who received a three-piece inflatable prosthesis compared with those who received a malleable prosthesis $(93.0 \%$ vs. $81.1 \%, P<0.05)$. Compared with patient satisfaction, spouse satisfaction was slightly lower, but this difference did not reach statistical significance $(P>0.05)$. Satisfaction between the types of prostheses did not differ based on the partner survey results.

\section{DISCUSSION}

Novel, effective pharmacological therapies have brought public attention to the medical management of ED. Oral inhibitors of phosphodiesterase type $\mathrm{V}$, penile injection therapy and vacuum erection devices are effective treatment options for men with ED, but these nonsurgical modalities do not work in severe cases. Under such circumstances, surgical management with PPI is the standard of care.

In this study, we report the use of PPI for the management of severe ED in Chinese patients. In our series, the average length of the left cavernosum was $16.0 \pm 1.5 \mathrm{~cm}$, and the average length of the right cavernosum was $16.1 \pm 1.5 \mathrm{~cm}$. These measurements are similar to data from a Korean population that we previously reported. ${ }^{6}$ In a study from Taiwan, China, the average cavernous length of 331 patients who underwent PPI was $17.2 \mathrm{~cm}$. It is apparent that the average cavernous length of Asian men may be less than what has been reported for men from Western countries (average: 19-22 cm); this disparity might be related to race or height differences. ${ }^{9}$ As prostheses used in China currently are manufactured based on data from Western men, small-sized cylinders suited to Asian men are not yet available.

PPI has a very favourable long-term satisfaction rate compared with oral pharmacotherapy and penile injections. ${ }^{11-13}$ The clinical efficacy of penile prostheses has been reported to be as high as $70 \%-96 \%,{ }^{7-10}$ similar to the results from our study. In our series, with a mean followup of 68.3 months, the mechanical failure rate was lower $(2.0 \%)$ than in prior reports. This finding may be a consequence of our patients' lower mean age ( $35.9 \pm 11.8$ years) due to the high proportion of young patients with primary ED (47.3\%) in our population. Additionally, given the greater economic burden of PPI faced by Chinese patients compared with men in Western countries, younger patients with a greater desire to maintain a healthy sexual relationship within their

Table 2 Patient and partner satisfaction post-penile prosthesis implantation, evaluated by Bhojwani's score

\begin{tabular}{lcc}
\hline Satisfaction score & Patients $(\mathrm{n}=201, \%)$ & Partners $(\mathrm{n}=201, \%)$ \\
\hline 10 & $38(18.9 \%)$ & $41(20.4 \%)$ \\
9 & $44(21.9 \%)$ & $37(18.4 \%)$ \\
8 & $43(21.4 \%)$ & $32(15.9 \%)$ \\
7 & $33(16.4 \%)$ & $27(13.4 \%)$ \\
6 & $21(10.4 \%)$ & $29(14.4 \%)$ \\
5 & $10(5.0 \%)$ & $14(7.0 \%)$ \\
4 & $6(3.0 \%)$ & $8(4.0 \%)$ \\
$1-3$ & $6(3.0 \%)$ & $13(6.5 \%)$ \\
\hline
\end{tabular}

marriages may have elected to pursue PPI at a greater rate than older patients with SED, who often choose not to pursue further treatments.

There are numerous potential complications of PPI surgery, including surgery-related complications (such as corpus cavernosum perforation, erosion and haematoma), infection (surgical field infection and prosthesis infection) and mechanical failure (such as leakage, connecting duct rupture, and spontaneous erection). ${ }^{14}$ Strict adherence to aseptic technique has been shown to reduce the incidence of postoperative infection from $12 \%$ to $4 \% .{ }^{15}$ A meta-analysis by Merino et al. ${ }^{16}$ showed lower infection rates for malleable prostheses compared with inflatable prostheses $(1.3 \%$ vs. $3.5 \%)$. Mechanical failure is more common in a multipiece inflatable prosthesis due to its complexity. The overall incidence of mechanical failure is approximately $5 \% .{ }^{17}$ The incidence of mechanical failure is significantly related to the length of follow-up time. ${ }^{18}$ Technical improvements have also reduced the incidence of mechanical failure. ${ }^{19-21}$

In our experience of 224 cases of PPI surgery, cross-perforation of the corpus cavernosa occurred in three cases of ED secondary to fibrosis. Successful implantation of a penile prosthesis was accomplished by insertion of a Hagar dilator into one corpus cavernosum, followed by implant insertion into the other corpus cavernosum. Infection is the most serious postoperative complication. In our experience, the incidence of infection is lower than in prior reports, likely due to the following factors: (i) strict disinfection of the operative area; (ii) preoperative antibiotic prophylaxis, with continuation of antibiotic administration for 3-7 days postoperatively; (iii) 1 week of hospitalisation for antibiotic therapy and care of the surgical site; and (iv) all cases were performed by an experienced surgeon. ${ }^{6}$

The IIEF-5, QoL and satisfaction scores were the primary parameters used to evaluate the efficacy of PPI. QoL was significantly improved by both types of prostheses. The IIEF-5 score is the most simple and convenient tool to assess ED, but it should be mentioned that the IIEF-5 score has not been validated to assess PPI. Ferguson et al. ${ }^{14}$ reported a postoperative improvement in QoL of 87\%, which is similar to our data.

We separately asked the patients and their partners to give a score from 1 to 10 to the result of the surgery. At the time of interview, $89.0 \%$ of patients and $82.5 \%$ of their partners indicated that PPI led to satisfying improvements in their sexual life. Of the $11 \%$ who were unsatisfied, the most common cause was insufficient penile length for normal intercourse. The satisfaction rates of the men were higher for three-piece prostheses $(93.0 \%)$ than for malleable prostheses $(81.1 \%)$ due to a lack of concealment and partners' sensation with malleable prostheses compared with three-piece prostheses. A meta-analysis published by Merino ${ }^{17}$ showed that average overall satisfaction with PPI was $83 \%$ (range: $55.9 \%-96.5 \%$ ). Unfortunately, a direct survey of 201/ 224 patients' partners was performed because numerous Chinese spouses declined to answer the questionnaire for cultural reasons.

These results suggested that PPI is a safe and effective treatment for ED in Chinese patients who fail management with medical therapy. PPI enhances sexual capacity and self-confidence and, through improved sexual function, improves the spousal relationship. PPI should be considered as a final choice for Chinese patients with SED refractory to medical management. An experienced surgeon perform PPI according to standard PPI procedure could reduce the postoperative rate of complications of PPI and could improve patient and partner's satisfaction ratio and QoL.

\section{AUTHOR CONTRIBUTIONS}

WDS, YMY and WSC assisted in the operations, performed statistical analyses and drafted the manuscript. YCZ, JL and LW participated in 
the patient follow-up. GYB and JP participated in sample processing and assisted in statistical analysis. ZCZ, BG and AKW participated in study coordination and helped draft the manuscript. ZCX, YLG and TFL performed the PPI surgery, monitored the study and helped draft the manuscript. All authors read and approved the final manuscript.

\section{COMPETING FINANCIAL INTERESTS}

The authors declared no competing financial interests.

\section{ACKNOWLEDGMENTS}

This work was supported by the National Natural Science Foundation of China (Nos. 81270693 and 81272531).

1 Lue TF. Erectile dysfunction. New Engl J Med 2000; 342: 1802-13.

2 Liu DF, Jiang H, Hong K, Zhao LM, Ma LL et al. [Epidemiological changes of ED patients: investigations in 11 Chinese cities during the past 5 years]. Zhonghua Nan Ke Xue 2009; 15: 724-6.

3 Hellstrom WJ, Gittelman M, Karlin G, Segerson T, Thibonnier M et al. Sustained efficacy and tolerability of vardenafil, a highly potent selective phosphodiesterase type 5 inhibitor, in men with erectile dysfunction: results of a randomized, doubleblind, 26-week placebo-controlled pivotal trial. Urology. 2003; 61: 8-14.

4 Hatzichristou DG, Apostolidis A, Tzortzis V, loannides E, Yannakoyorgos K et al. Sildenafil versus intracavernous injection therapy: efficacy and preference in patients on intracavernous injection for more than 1 year. J Urol 2000; 164: 1197-200.

5 Mulcahy JJ, Austoni E, Barada JH, Choi HK, Hellstrom WJ et al. The penile implant for erectile dysfunction. J Sex Med 2004; 1: 98-109.

6 Xin ZC, Guo YL, Choi HK. A retrospective study of 548 cases of erectile dysfunction treated by penile prosthesis implantation. Chin J Urol 2005; 21: 755-7.
7 Song WD, Wu YG, Yuan YM, Peng J, Zhang ZC et al. One-stage direct-vision internal urethrotomy and penile prosthesis implantation for severe erectile dysfunction with urethral stricture. Chonnam Med J 2009; 45: 33-7.

8 Bhojwani AG, Jain R, Kockelbergh RC, Terry TR. Sexual satisfaction after penile prosthesis insertion for the treatment of erectile dysfunction. Sex Dysfuct 1998; I: 133-6.

9 Zhou F, Xin H, Liu T, Li GY, Gao ZZ et al. Effects of Icariside II on improving erectile function in rats with streptozotocin-induced diabetes. J Androl 2012; 33: 832-44.

10 Chiang HS, Wu CC, Wen TC. 10 years of experience with penile prosthesis implantation in Taiwanese patients. J Urol 2000; 163: 476-80.

11 Carson CC, Mulcahy JJ, Govier FE. Efficacy, safety and patient satisfaction outcomes of the AMS 700CX inflatable penile prosthesis: results of a long-term multicenter study. AMS 700CX Study Group. J Urol 2000; 164: 376-80.

12 Zhu YC, Zhao JL, Wu YG, Yuan YM, Lian WQ et al. Clinical features and treatment options for Chinese patients with severe primary erectile dysfunction. Urol 2010; 76: 387-90.

13 Rajpurkar A, Dhabuwala CB. Comparison of satisfaction rates and erectile function in patients treated with sildenafil, intracavernous prostaglandin $\mathrm{E} 1$ and penile implant surgery for erectile dysfunction in urology practice. J Urol 2003; 170: 159.

14 Ferguson KH, Cespedes RD. Prospective long-term results and quality-of-life assessment after Dura-II penile prosthesis placement. Urology 2003; 61: 437-41.

15 Sadeghi-Nejad $\mathrm{H}$. Penile prosthesis surgery: a review of prosthetic devices and associated complications. J Sex Med 2007; 4: 296-309.

16 Minervini A, Ralph DJ, Pryor JP. Outcome of penile prosthesis implantation for treating erectile dysfunction: experience with 504 procedures. BJU Int 2005; 97 129-33.

17 Merino A. Penile prosthesis for the treatment of erectile dysfunction. Actas Urol Esp 2006; 30: 159-69.

18 Munoz JJ, Ellsworth PI. The retained penile prosthesis reservoir: a risk. Urology. 2000; 55: 949.

19 Randrup ER. Clinical experience with 180 inflatable penile prostheses. South Med J 1995; 88: 47-51.

20 Milbank AJ, Montague DK, Angermeier KW, Lakin MM, Worley SE. Mechanical failure of the American Medical Systems UItrex inflatable penile prosthesis: before and after 1993 structural modification. J Urol 2002; 167: 2502-6.

21 Mansouri MD, Boone TB, Darouiche RO. Comparative assessment of antimicrobial activities of antibiotic-treated penile prostheses. Eur Urol 2009; 56: 1039-46. 